\title{
Comprehensive Noise Analysis in PPG Read-out Chains
}

\author{
Antonino Caizzone, Assim Boukhayma and Christian Enz \\ École Polytechnique Fédérale de Lausanne (EPFL) - Integrated Circuit Laboratory (ICLAB), Neuchâtel, Switzerland \\ antonino.caizzone@epfl.ch
}

\begin{abstract}
This paper presents an analytical comparison of two commonly used read-out structures for pulse oximeters, in a wearable application context. The objective is to analyze and highlight the trade-off between the SNR and the power consumption. The proposed analysis clarifies, by the means of analytical equations, the impact of the input photocurrent, the photodiode parasitic capacitance, the feedback impedance and the system bandwidth on the SNR of the two proposed front-end topologies. The paper shows that using a charge-amplifier in the front-end requires 8 times less LED power than a transresistance amplifier for achieving the same SNR.
\end{abstract}

Keywords-Noise, PPG, pulse oximeter, ZTIA, CTIA, CDS, shot, flicker, thermal, quantization.

\section{INTRODUCTION}

The need for mobile heath care systems and the increased interest in fitness and wellness is calling for more affordable, precise and reliable wearable health monitoring devices. In this context, photoplethysmography (PPG) appears to be a key technology allowing non-invasive monitoring of vital biological indicators such as heart rate, blood oxygen saturation, respiration rate and arterial pressure. The power consumption of PPG systems is limited by the active light source emission (LED). However, the LED power can be reduced provided the noise floor of the font-end electronics is decreased proportionally to achieve the same signal-to-noise ratio (SNR).

In this paper we present a detailed analysis of the main noise sources in PPG read-out chains, including the shot noise associated with the input light and the thermal, flicker and quantization noise of the read-out chain. The analysis is performed on the two commonly used front-end topologies: the first is based on a transimpedance amplifier with RC feedback (ZTIA) [1], [2], while the second on a (charge) transimpedance amplifier with capacitive feedback (CTIA) [1], [3], [4]. The proposed analysis clarifies, by the means of analytical equations the impact of the input photocurrent, the photodiode parasitic capacitance, the feedback impedance and the system bandwidth (BW) on the SNR of the two proposed front-end topologies. This allows to directly determine the minimum LED power for a targeted minimum SNR $(28.5 \mathrm{~dB})$ [5] and a set of design parameters.

The paper is organized as follows: Section II introduces the main noise sources analyzed in the work. Section III focuses on the SNR vs LED power comparison between the two proposed structures and Section IV concludes the paper.

\section{NOISE SOURCES}

As shown in Fig. 1, the PPG signal is obtained by shining light from LEDs at different wavelengths, usually red and infrared, into a human tissue, e.g. finger, forehead, ear lobs etc. The LED is duty cycled in order to save power: typically LEDs are made shining for a duration ranging from the $1 \%$ to the $10 \%$ of their full period. A photodetector (PD) detects the light transmitted through or reflected from the tissue and transforms it into a photogenerated current.

The detected signal, i.e. PPG, consists of two different components: a large DC (static) component corresponding to the light diffusion through tissues and non-pulsatile blood layers, and a small AC (pulsatile) due to the diffusion through the arterial blood. The AC component is only a small fraction (typically $0.2 \%$ to $2 \%$ ) of the DC one [6].

The PD also absorbs ambient light photons whose associated photocurrent has to be compensated in the read-out chain. The simplest way to do it is to perform Correlated Double-Sampling (CDS): an ambient sample is taken, which is then subtracted from the LED sample, the PPG signal. If the time between the two samples is comparable with the LED pulse time width, $T_{O N}$, an (almost) ambient light free PPG signal is obtained.

Typical CDS is performed by the means of a sample-andhold $(\mathrm{S} / \mathrm{H})$ stage. The CDS has an important impact on the noise. Indeed, it cancels the offset, e.g. the ambient light, and dramatically reduces low frequency noise, such as the flicker noise. The CDS transfer function can be expressed as [7]

$$
\left|H_{C D S}(f)\right|^{2}=4 \sin ^{2}\left(\pi T_{C D S} f\right)
$$

where $T_{C D S}$ is the time between two consecutive CDS samples. In the following we consider $T_{C D S}=T_{O N}$. The transfer function in (1) actually corresponds to a high-pass filter for the noise, strongly reducing the low frequency component.

In the following analysis the PD shunt resistance, $R_{P D}$, can usually be neglected since typically large enough to be considered as an open circuit. The overall noise variances contributions will be evaluated at the output of the analog-todigital converter (ADC) and eventually used to compare the two proposed structures in terms of SNR vs photogenerated current, $I_{p h}$. Note that $I_{p h}$ is roughly linearly proportional to the LED current, i.e. LED power, through a constant representing the tissue attenuation, whose values range from $10^{-3}$ to $10^{-4}$ [5]. 


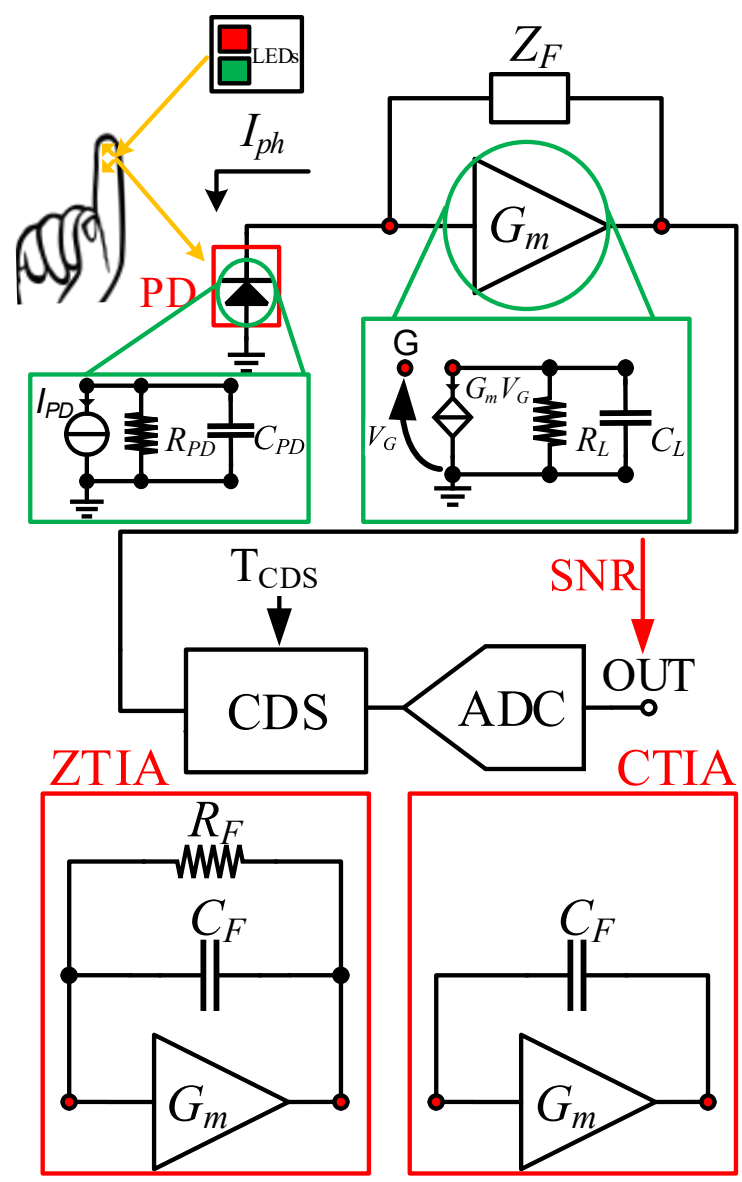

Fig. 1. Schematic of a classical PPG read-out chain showing the two possible alternative implementations, i.e. ZTIA-based or CTIA-based

For a generic noise source, having a Power-Spectral Density (PSD) $S_{n}(f)$, its corresponding noise variance, accounting for the effect of CDS, is equal to

$$
\sigma_{n}^{2}=\int_{0}^{\infty} S_{n}(f) \cdot\left|H_{n}(f)\right|^{2} \cdot\left|H_{C D S}(f)\right|^{2} d f
$$

where $H_{n}(f)$ and $H_{C D S}(f)$ are the noise transfer function and the CDS transfer function, given in (1), respectively.

\section{A. Shot Noise}

The shot noise results from the discrete nature of the LED photon flux and appears at the input of the read-out chain. Eq. (3) reports the current PSD associated with the shot noise, for a photogenerated current

$$
S_{I_{s h}^{2}}=2 q I_{p h}
$$

where $q$ is the charge of an electron, $1.6 \times 10^{-19} \mathrm{C}$, and $I_{p h}$ is the (DC) photogenerated current, as shown in Fig. 1. As far as the shot noise is concerned, from now on the analysis will be split for the ZTIA and CTIA cases, independently.
1) ZTIA: Since the shot noise current appears in parallel to the photocurrent source, the noise transresistance is identical to the signal transresistance

$$
H_{n, s h_{Z T I A}}(f)=R_{F} \cdot \frac{1-j f / f_{z_{s h}}}{\left(1+j f / f_{p 1}\right)\left(1+j f / f_{p 2}\right)},
$$

where $f_{p 1}=\frac{G_{m}}{2 \pi\left(C_{P D}+C_{F} G_{m} R_{F}\right)}, f_{p 2}=\frac{\left(C_{P D}+C_{F} G_{m} R_{F}\right)}{2 \pi C_{F} C_{P D} R_{F}}$ and $f_{z_{s h}}=\frac{G_{m}}{2 \pi C_{F}}$. For noise calculations the noise transresistance (4) can be approximated by a first order response, whose cut-off frequency is $f_{p 1}$. Indeed, in practical cases $f_{p 1} \ll f_{p 2} \ll f_{z_{s h}}$. Using (2), the shot noise variance for the ZTIA case becomes

$$
\sigma_{s h_{Z T I A}}^{2} \cong \alpha_{s h_{Z T I A}} \cdot 2 q I_{p h} \cdot B W_{n, s h_{Z T I A}} \cdot R_{F}^{2},
$$

where $B W_{n}$ defines the equivalent noise $\mathrm{BW}$, that, for the ZTIA shot noise case, is approximately equal to

$$
B W_{n, s h_{Z T I A}} \cong \frac{\pi}{2} f_{p 1}
$$

and $\alpha_{s h_{Z T I A}}$ is an unitless circuit design parameter, accounting for the effect of CDS, given by

$$
\alpha_{s h_{Z T I A}}=\frac{1}{B W_{n, s h_{Z T I A}}} \int_{0}^{\infty} \frac{4 \sin ^{2}\left(\pi T_{O N} f\right)}{1+\left(f / f_{p 1}\right)^{2}} d f \cong 2 .
$$

This integral has been numerically calculated and is simply equal to 2 , when $2 \pi T_{O N} f_{p 1}>5$ (which is the condition for sufficient settling of the signal) [8]. Indeed, the shot noise behaves as a white noise. Hence, the CDS corresponds to the differentiation of two uncorrelated noise samples, which results in doubling the noise variance.

2) CTIA: Unlike the ZTIA case, in the case of the CTIA, the PSD of the shot noise gets fully integrated by the feedback capacitance, $C_{F}$. The shot noise transresistance of the CTIA is

$$
H_{n, s h_{C T I A}}(f)=\frac{1}{j 2 \pi f C_{F}} \frac{1-j f / f_{z_{s h}}}{1+j f / f_{p}},
$$

where $f_{z_{s h}}$ and $f_{p}$ are the zero and the pole (signal BW), respectively, related to the shot noise transresistance. For noise calculations the transresistance in (8) can be approximated by a first order response, whose cut-off frequency is given by

$$
f_{p}=G_{m} / 2 \pi C_{P D}
$$

Using (2), the shot noise variance for the CTIA case becomes

$$
\sigma_{s h_{C T I A}}^{2} \cong \alpha_{s h_{C T I A}} \cdot 2 q I_{p h} T_{O N} \cdot \frac{1}{C_{F}^{2}},
$$

where $\alpha_{s h_{C T I A}}$ is

$$
\alpha_{s h_{C T I A}}=\frac{1}{T_{O N}} \int_{0}^{\infty} \frac{1}{(2 \pi f)^{2}} \cdot \frac{4 \sin ^{2}\left(\pi T_{O N} f\right)}{1+\left(\frac{f}{f_{p}}\right)^{2}} d f \cong \frac{1}{2}
$$

This integral has been numerically calculated and is simply equal to $1 / 2$, when $2 \pi T_{O N} f_{p}>5$ [8].

Thus, the shot noise for the CTIA case corresponds to a Wiener process for which the variance increases linearly with $T_{O N}$ [9], as shown in (10). 


\section{B. Thermal Noise}

In a PPG read-out chain the thermal noise originates both from the MOS channels and the circuit resistors. Eq. (12) shows (from left to right) the thermal noise current PSD of a MOS transistor and a generic resistor

$$
S_{I_{c h, t h}^{2}}=4 k T \gamma G_{m} \quad S_{I_{R, t h}^{2}}=4 k T / R,
$$

where $k$ is the Boltzmann constant, $T$ the temperature in Kelvin, $\gamma \triangleq G_{m} R_{n}$ is the thermal noise excess factor related to the saturated transistor $\left(R_{n}\right.$ is the input referred thermal noise resistance) [10], $G_{m}$ the transconductance of the transconductor and $R$ the value of the resistor. Note that $\gamma$ increases with the number of saturated transistors used in the OTA, i.e. "Operational Transconductance-Amplifier". As for the shot noise, the following analysis will be separated between the ZTIA and the CTIA cases, independently.

1) ZTIA: Regarding the ZTIA, two thermal noise contributions should be taken into account: the one related to the MOS channel and the one coming from the feedback resistance, $R_{F}$. Nevertheless, the noise variance related to the channel dominates over the feedback resistance's, which means that the latter will be neglected. For the channel thermal noise the corresponding noise transresistance is

$$
H_{n, t h_{Z T I A}}(f)=\frac{1}{G_{m}} \cdot \frac{1+j f / f_{z_{t h}}}{\left(1+j f / f_{p 1}\right)\left(1+j f / f_{p 2}\right)},
$$

where $f_{z_{t h}}=\frac{1}{2 \pi R_{F}\left(C_{F}+C_{P D}\right)}$. From (2), the channel thermal noise variance for the ZTIA case becomes

$$
\sigma_{t h_{Z T I A}}^{2} \cong \alpha_{t h_{Z T I A}} \cdot \frac{4 k T \gamma}{G_{m}} \cdot B W_{n, t h_{Z T I A}}
$$

where $\alpha_{t h_{Z T I A}}$ is

$$
\alpha_{t h_{Z T I A}}=\frac{1}{B W_{n, t h_{Z T I A}}} \int_{0}^{\infty} \frac{4 \sin ^{2}\left(\pi T_{O N} f\right)\left[1+\left(\frac{f}{f_{z_{t h}}}\right)^{2}\right]}{\left[1+\left(\frac{f}{f_{p 1}}\right)^{2}\right]\left[1+\left(\frac{f}{f_{p 2}}\right)^{2}\right]} d f \cong 2,
$$

and $B W_{n, t h_{Z T I A}}$ is

$$
B W_{n, t h_{Z T I A}} \cong \frac{\pi}{2} f_{p 2} \cdot \frac{\left(C_{P D}+C_{F}\right)^{2}\left(G_{m} R_{F}\right)^{2}}{\left(C_{P D}+C_{F} G_{m} R_{F}\right)^{2}},
$$

considering $f_{z_{t h}} \ll f_{p 1} \ll f_{p 2}$.

So far we have shown that, no matter the PSD source, the CDS simply doubles the thermal noise variance. This result is expected since, as far as the thermal noise is concerned, the CDS subtracts two samples which are uncorrelated. We will see that the same considerations apply for the CTIA case.

2) CTIA: Regarding the CTIA based circuit, the only thermal noise contribution is the one related to the channel, whose transresistance is

$$
H_{n, t h_{C T I A}}(f)=\frac{1}{G_{m}} \cdot \frac{C_{P D}+C_{F}}{C_{F}} \cdot \frac{1}{1+j f / f_{p}},
$$

where $f_{p}$ is the pole (signal $\mathrm{BW}$ ) related to the channel thermal noise transresistance. Using (2), the thermal noise variance for the CTIA case becomes

$$
\sigma_{t h_{C T I A}}^{2} \cong \alpha_{t h_{C T I A}} \cdot \frac{4 k T \gamma}{G_{m}} \cdot B W_{n, t h_{C T I A}}
$$

where $\alpha_{t h_{C T I A}} \cong 2$ (same calculation as in $\alpha_{s h_{Z T I A}}$ ) and $B W_{n, t h_{C T I A}}$ is

$$
B W_{n, t h_{C T I A}}=\frac{\pi}{2} f_{p} \cdot\left(\frac{C_{P D}+C_{F}}{C_{F}}\right)^{2} .
$$

\section{Flicker Noise}

The current PSD of the MOS transistor for the flicker noise case is defined as

$$
S_{I_{1 / f}^{2}}=K_{F} G_{m}^{2} /\left(C_{o x}^{2} W L f\right),
$$

where $K_{\mathrm{F}}$ is the flicker (technological) noise parameter and $C_{o x}$ the gate oxide capacitance per unit area. For a $180 \mathrm{~nm}$ process $K_{\mathrm{F}}=1 \cdot 10^{-27}\left[\frac{\mathrm{J} \cdot \mathrm{F}}{\mathrm{m}^{2}}\right]$ and $C_{o x}=8.46 \cdot 10^{-3}\left[\frac{\mathrm{F}}{\mathrm{m}^{2}}\right]$.

1) ZTIA: Regarding the ZTIA circuit, the flicker noise transresistance is the same of the channel thermal noise, (13). Using (2), the flicker noise variance for the ZTIA case becomes

$$
\sigma_{1 / f_{Z T I A}}^{2} \cong \alpha_{1 / f_{Z T I A}} K_{F} /\left(C_{o x}^{2} W L\right)
$$

where $\alpha_{1 / f_{Z T I A}}$ is

$\alpha_{1 / f_{Z T I A}}=\int_{0}^{\infty} \frac{1}{f} \frac{4 \sin ^{2}\left(\pi T_{O N} f\right)\left[1+\left(\frac{f}{f_{z_{t h}}}\right)^{2}\right]}{\left[1+\left(\frac{f}{f_{p 1}}\right)^{2}\right]\left[1+\left(\frac{f}{f_{p 2}}\right)^{2}\right]} d f \cong 4.5$

under the assumption of $f_{z_{t h}} \ll f_{p 1} \ll f_{p 2} . \alpha_{1 / f_{Z T I A}}$ is the CDS parameter, increasing (logarithmically) with the ratio between $T_{C D S}$ and the signal settling-time. Assuming that $T_{C D S}$ is roughly equal to the settling time, $\alpha_{1 / f_{Z T I A}}$ is, as shown by (22), approximately equal to 4.5 .

2) CTIA: As for the ZTIA case the 1/f noise shares the same transresistance of the channel thermal noise, (17). The flicker noise variance for the CTIA is given by

$$
\sigma_{1 / f_{C T I A}}^{2} \cong \alpha_{1 / f_{C T I A}} \frac{K_{F}}{C_{o x}^{2} W L}\left(\frac{C_{P D}+C_{F}}{C_{F}}\right)^{2},
$$

where $\alpha_{1 / f_{C T I A}}$ is

$$
\alpha_{1 / f_{C T I A}}=\int_{0}^{\infty} \frac{1}{f} \cdot \frac{4 \sin ^{2}\left(\pi T_{O N} f\right)}{1+\left(\frac{f}{f_{p}}\right)^{2}} d f \cong 4.5 .
$$

\section{Quantization Noise}

The variance due to the quantization process in the ADC is given by

$$
\sigma_{A D C}^{2}=\Delta^{2} / 12
$$

where, in (25), $\Delta$ is the quantization step (assuming uniform quantization) which depends on the reference voltage $V_{\text {ref }}$ and the resolution $N_{b i t}$ and typically ranges from $50 \mu V_{r m s}$ to 150 $\mu V_{\text {rms }}$ [11]. 


\section{DISCUSSION}

This section compares the two front-end structures, i.e. ZTIA and CTIA, in terms of SNR, targeting a minimum SNR of $28.5 \mathrm{~dB}$ for an accuracy within $2 \%$ of the $\mathrm{S}_{p} \mathrm{O}_{2}$ (oxygensaturation) corresponding signal [5]. The SNR at the output of the ADC can be expressed as

$$
S N R=10 \log _{10} \frac{S^{2}}{N^{2}}=10 \log _{10} \frac{V_{o u t}^{2}}{\sigma_{s h}^{2}+\sigma_{t h}^{2}+\sigma_{1 / f}^{2}+\sigma_{A D C}^{2}} .
$$

The SNR comparison between the ZTIA and the CTIA is performed assuming the same signal bandwidth

$$
f_{p}=f_{p 1}=B W=\frac{1}{2 \pi \tau} .
$$

\section{A. ZTIA}

The output voltage in the ZTIA case is given by

$$
V_{\text {out }}=\eta R_{F} I_{p h},
$$

where $\eta$ is the signal contrast, i.e. $\frac{A C}{D C}$. In this work $\eta$ has been considered equal to $0.2 \%$ in order to account for the worst case (perfusion) condition.

\section{B. CTIA}

The output voltage in the CTIA case is given by

$$
V_{\text {out }}=\eta T_{O N} I_{p h} / C_{F},
$$

where $T_{O N}$ is the ON-time of the LED (LED time duration).

\section{Comparison}

Fig. 2 shows the SNR of both circuit topologies as function of the average input photocurrent, i.e. LED power, for different PD parasitic capacitances, pointing out the shot noise and the electronic read noise limited regions. The analysis has been performed based on the parameters given in Table I (these parameters are related to $C_{P D}=100 \mathrm{pF}$ ). Assuming full shot noise limitation, i.e. large $I_{p h}$ condition, the ratio between the two SNR is

$$
\frac{S N R_{C T I A}}{S N R_{Z T I A}} \cong 2 \pi \cdot B W \cdot T_{O N}=\frac{T_{O N}}{\tau} .
$$

$2 \pi \cdot B W \cdot T_{O N}$ must be much larger than 1 to ensure enough signal settling. The condition to ensure efficient signal settling is $2 \pi \cdot B W \cdot T_{O N} \cong 10$. This means that the SNR of the CTIA based configuration is at least 10 times better than the ZTIA based one. Indeed, Fig. 2 shows exactly $10 \mathrm{~dB}$ difference between the two cases in the shot noise dominated region.

TABLE I

DESIGN PARAMETERS

\begin{tabular}{c|c||c|c}
\hline Parameter & Value & Parameter & Value \\
\hline$G_{m_{C T I A}}$ & $10 \mu S$ & $T_{O N}$ & $100 \mu \mathrm{s}$ \\
$G_{m_{Z T I A}}$ & $100 \mu S$ & $R_{L}$ & $100 \mathrm{M} \Omega$ \\
$R_{F}$ & $1 M \Omega$ & $W$ & $5 \mu \mathrm{m}$ \\
$C_{F}$ & $9 p F$ & $L$ & $2 \mu \mathrm{m}$ \\
$C_{L}$ & $1 p F$ & $\Delta$ & $100 \mu V_{R M S}$ \\
\hline
\end{tabular}

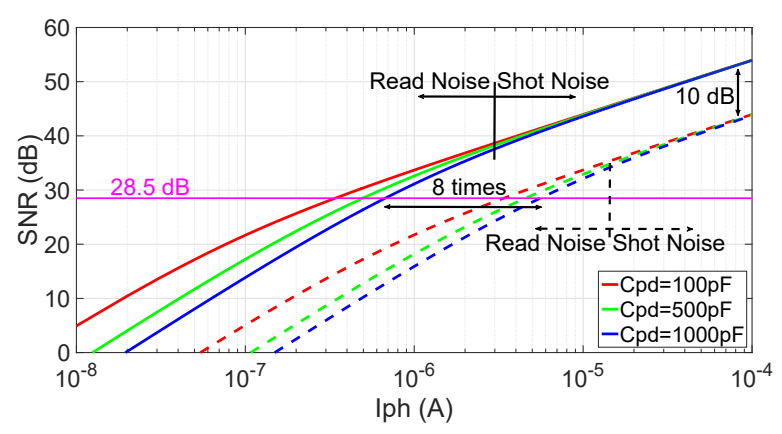

Fig. 2. SNR of both CTIA (solid line) and ZTIA (dashed line) based design versus the input photocurrent, for three different PD parasitic capacitance values

\section{CONCLUSION}

This work compares two implementations of a PPG read-out chain, one using a transimpedance amplifier (ZTIA) and the other a charge amplifier (CTIA). It is shown that for achieving the same SNR, assuming an identical signal bandwidth, a ZTIAbased read-out chain comes with both larger LED power ( 8 times more) and a larger $G_{m}$, compared to a CTIA-based one. This advantage is attributed to the integration feature of the CTIA. Indeed, the CTIA comes intrinsically with a larger BW than the ZTIA. Comparing the two for the same BW means, in other words, having a larger signal gain for the CTIA than the ZTIA. Moreover the ZTIA shows a larger read noise than the CTIA, which is, on the contrary, intrinsically more shot noise limited. Notice that for the target SNR, both solutions show still an important electronic read noise level, calling for further design optimizations.

\section{REFERENCES}

[1] P. Schönle, et al., "A Power-Efficient Multi-Channel PPG ASIC with 112 dB Receiver DR for Pulse Oximetry and NIRS," CICC, 2017.

[2] A. K. Y. Wong, K. P. Pun, Y. T. Zhang, and K. N. Leung, "A Low-Power CMOS Front-End for Photoplethysmographic Signal Acquisition With Robust DC Photocurrent Rejection," IEEE Transactions on Biomedical Circuits and Systems, vol. 2, no. 4, pp. 280-288, Dec 2008.

[3] E. S. Winokur, T. O'Dwyer, and C. G. Sodini, "A Low-Power, DualWavelength Photoplethysmogram (PPG) SoC With Static and TimeVarying Interferer Removal," IEEE Transactions on Biomedical Circuits and Systems, vol. 9, no. 4, pp. 581-589, Aug 2015.

[4] S. V. Gubbi and B. Amrutur, "Adaptive Pulse Width Control and Sampling for Low Power Pulse Oximetry," IEEE Transactions on Biomedical Circuits and Systems, vol. 9, no. 2, pp. 272-283, April 2015.

[5] K. N. Glaros and E. M. Drakakis, "A Sub-mW Fully-Integrated Pulse Oximeter Front-End," IEEE Transactions on Biomedical Circuits and Systems, vol. 7, no. 3, pp. 363-375, June 2013.

[6] J. G. Webster, Design of Pulse Oximeters. Bristol, PA, USA: Philadelphia: Institute of Physics Pub., 1997.

[7] C. Enz and A. Boukhayma, "Recent Trends in Low-Frequency Noise Reduction Techniques for Integrated Circuits." Noise and Fluctuations (ICNF), 2015 International Conference on, pp. 1-6, 2015.

[8] A. Boukhayma, A. Peizerat, and C. Enz, "Noise Reduction Techniques and Scaling Effects Towards Photon Couting CMOS Image Sensors." Sensors, vol. 16, no. 4, p. 514, April 2016.

[9] A. Papoulis, Probability, Random Variables, and Stochastic Processes. Mc-Graw Hill, 1984

[10] C. Enz and E. A. Vittoz, Charge-Based MOS Transistor Modeling: The EKV model for low-power and RF IC design. John Wiley \& Sons, Ltd, 2006.

[11] T. I. (Data-Sheet), "ADS111x Ultra-Small, Low Power, $\mathrm{I}^{2} \mathrm{C}-\mathrm{Compatible,}$ 860-SPS,16-Bit ADCs." 